Check for updates

Cite this: Phys. Chem. Chem. Phys., 2021, 23, 22057

Received 7th June 2021 Accepted 15th September 2021 DOI: $10.1039 / \mathrm{d} 1 \mathrm{cp} 02537 f$

rsc.li/pccp

\section{Rovibronic spectroscopy of PN from first principles $\dagger$}

\author{
Mikhail Semenov, (D) ab Nayla El-Kork, (D) ${ }^{c}$ Sergei N. Yurchenko (D) *a and \\ Jonathan Tennyson (iD) a
}

\begin{abstract}
We report an ab initio study on the rovibronic spectroscopy of the closed-shell diatomic molecule phosphorous mononitride, PN. The study considers the nine lowest electronic states, $X^{1} \Sigma^{+}, A{ }^{1} \Pi, C^{1} \Sigma^{-}$, $D^{1} \Delta, E{ }^{1} \Sigma^{-}, a{ }^{3} \Sigma^{+}, b^{3} \Pi, d^{3} \Delta$ and $e^{3} \Sigma^{-}$using high level electronic structure theory and accurate nuclear motion calculations. The ab initio data cover 9 potential energy, 14 spin-orbit coupling, 7 electronic angular momentum coupling, 9 electric dipole moment and 8 transition dipole moment curves. The Duo nuclear motion program is used to solve the coupled nuclear motion Schrödinger equations for these nine electronic states and to simulate rovibronic absorption spectra of ${ }^{31} \mathrm{p}^{14} \mathrm{~N}$ for different temperatures, which are compared to available spectroscopic studies. Lifetimes for all states are calculated and compared to previous results from the literature. The calculated lifetime of the $A^{1} \Pi$ state shows good agreement with an experimental value from the literature, which is an important quality indicator for the ab initio A-X transition dipole moment.
\end{abstract}

\section{Introduction}

Phosphorus is considered to be one of the key elements as a source of life and replication on our planet, ${ }^{1,2}$ with PN being one of the candidates in star and meteorite evolution to provide the necessary life building material. There have been multiple observations of PN in different media in space: hot dense molecular clouds, ${ }^{3,4}$ energetic star forming regions, ${ }^{5,6}$ cold cloud cores, ${ }^{5,7}$ red giant stars $^{8-10}$ and protoplanetary nebula. ${ }^{8}$ Regardless of high astrophysical and astrobilogical importance, phosphorous mononitride is one of the experimentally least-well studied diatomic molecules of its isoelectronic group $\left(\mathrm{P}_{2}, \mathrm{SiO}, \mathrm{N}_{2}, \mathrm{CS}\right)$.

The molecule was reported for the first time by Curry et al. ${ }^{11}$ using a spectroscopic study. Many other subsequent spectroscopic studies have taken place including photoelectron, ${ }^{12}$ fluorescence, ${ }^{13}$ matrix infrared,${ }^{14}$ microwave ${ }^{15-17}$ and Fourier Transform Infra-Red (FTIR). ${ }^{18}$ Most of the high resolution spectroscopy experiments with PN concentrated on the electronic system A ${ }^{1} \Pi-\mathrm{X}^{1} \Sigma^{+},{ }^{11,13,19-21}$ with $\mathrm{E}{ }^{1} \Sigma^{+}-\mathrm{X}{ }^{1} \Sigma^{+}$being confirmed afterwards, ${ }^{22-24}$ and several valence and Rydberg states have been studied as well. ${ }^{25,26}$

Measured lifetimes can be important indicators of intensities and Einstein A coefficients. So far only one experimental

\footnotetext{
${ }^{a}$ Department of Physics and Astronomy, University College London, Gower Street, WC1E6BT London, UK. E-mail: s.yurchenko@ucl.ac.uk

${ }^{b}$ Department of Science and Research, Moscow Witte University,

2nd Kozhukhovskiy Passage, Moscow, Russian Federation

'Department of Physics, Khalifa University, Abu Dhabi, United Arab Emirates

$\dagger$ Electronic supplementary information (ESI) available. See DOI: 10.1039/ d1cp02537f
}

work reports lifetime measurements for the $\mathrm{A}^{1} \Pi$ state of PN molecule $^{27}$ using Hanle effect, with several $a b$ initio works providing computed values for PN lifetimes. ${ }^{28,29}$

The mass spectrometric experiment by Gingeric ${ }^{30}$ reports PN's dissociation energy $D_{0}$ to be $6.35 \pm 0.22 \mathrm{eV}$, which is lower than experimental $D_{0}$ values by Huffman et al. ${ }^{31}$ and Uy et al., ${ }^{32}$ $7.1 \pm 0.05 \mathrm{eV}$ and $7.57 \pm 0.03 \mathrm{eV}$, respectively. In a combination of a high-level $a b$ initio and microwave spectroscopy study, Cazzoli et al. ${ }^{17}$ suggested a $D_{0}$ value of $6.27 \mathrm{eV}$. This is close both to the originally predicted value by Curry et $a l .{ }^{33}$ and to the experimental value of Gingeric. ${ }^{30}$

Several theoretical investigations of PN are available in the literature. The most recent was carried out by Qin et al. ${ }^{29}$ who reported spectroscopic constants for the lowest five singlet $\left(\mathrm{X}{ }^{1} \Sigma^{+}, \mathrm{A}{ }^{1} \Pi, \mathrm{D}{ }^{1} \Delta, \mathrm{C}^{1} \Sigma^{-}, 2{ }^{1} \Pi\right.$ ), six triplet (including a ${ }^{3} \Sigma^{+}$, b ${ }^{3} \Pi, \mathrm{d}^{3} \Delta, \mathrm{e}^{3} \Sigma^{-}$) and two quintet electronic states of PN. In that paper all the states were studied at the internally contracted multi-reference configuration interaction (icMRCI) level of theory with Davidson correction (+Q). Similarly, Abbiche et al. ${ }^{34}$ reported an ab initio study of seven states of $\mathrm{PN}, \mathrm{X}^{1} \Sigma^{+}, \mathrm{A}^{1} \Pi, \mathrm{D}^{1} \Delta$, $\mathrm{C}^{1} \Sigma^{-}, 2{ }^{1} \Pi, \mathrm{E}{ }^{1} \Sigma^{+}, 3{ }^{1} \Sigma^{+}$, as well as of 10 triplet and 3 quintet electronic states. The main purpose of their study was to interpret perturbation and predissociation effects in the observed transitions.

In this work we present a comprehensive ab initio spectroscopic model for the nine lowest electronic states of phosphorous mononitride, $\mathrm{X}^{1} \Sigma^{+}$, A ${ }^{1} \Pi, \mathrm{C}^{1} \Sigma^{-}$, D ${ }^{1} \Delta, \mathrm{E}^{1} \Sigma^{+}, \mathrm{a}^{3} \Sigma^{+}, \mathrm{b}{ }^{3} \Pi, \mathrm{d}{ }^{3} \Delta$, e ${ }^{3} \Sigma^{-}$, consisting of potential energy curves (PECs), transition dipole moments curves (TDMCs), spin-orbit coupling curves (SOCs) and angular momentum coupling curves (AMCs) using 
the icMRCI + Q method and calculate the rovibronic energies and transition probabilities as an ab initio line list for PN. Producing such line lists for molecules of astrophysical significance is one of the main objectives of the ExoMol project. ${ }^{35}$ These curves, with some simple adjustment of the minimum energies of the PECs, are used to solve the coupled nuclearmotion Schrödinger equation with the program Duo. ${ }^{36}$ The spectroscopic model and ab initio curves are provided as part of the supplementary material. Our open source code Duo can be accessed via http://exomol.com/software/. The results of Duo calculation are then used to generate rovibronic spectra of PN and compare to systems previously reported in the literature.

\section{Computational details}

\subsection{Ab initio calculations}

Using MOLPRO 2020, ${ }^{37}$ ab initio calculations were performed for nine low-lying electronic states of PN. Apart from PECs, we

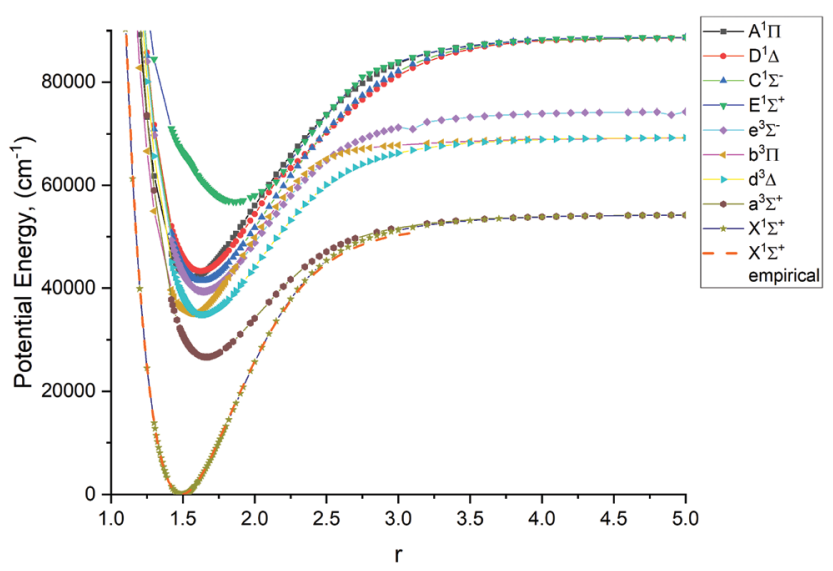

Fig. 1 Ab initio (icMRCl + Q/ECP10MWB) PECs of PN: The lowest 5 singlets and 4 lowest triplets. also computed (T)DMCs, SOCs and EAMCs. For the ab initio calculations we used the icMRCI $\operatorname{method}^{38}$ in conjunction with the effective-core-potential (ECP) method ECP10MWB (Stuttgart/ Cologne) for phosphorus ${ }^{39}$ and aug-cc-pCV5Z ${ }^{40}$ basis set for nitrogen. The initial complete active space self-consistent field (CASSCF) calculation over which the configuration interaction calculations were built was for the $\mathrm{X}^{1} \Sigma^{+}$state only. In conjunction with ECP for phosphorus, the active space was selected to be $(6,2,2,0)$ with $(1,0,0,0)$ closed orbitals. The state averaging set contained 96 states: 24, 24, 24 and 24 states of the singlets, triplets, pentets and septets. This level of theory will be referenced to as icMRCI + Q/ECP10MWB.

The calculations of the spin-orbit couplings were too difficult to perform for the icMRCI + Q/ECP10MWB level of theory, taking too long to complete and producing wrong data. We therefore decided to use a non-ECP level of theory for our spin-orbit calculations. To this end, we selected the $a b$ initio level of theory similar to that used by Qin et $a l .{ }^{29}$ with an active space of $(9,3,3,0)$. In this case the state averaging set consisted of 11 singlet configurations $\left(4 \mathrm{~A}_{1}, 2 \mathrm{~B}_{1}, 2 \mathrm{~B}_{2}\right.$ and $\left.3 \mathrm{~A}_{2}\right)$. The Douglass-Kroll correction was taken into account with or without core-valence correlation. These levels of theory will be referenced to as icMRCI/aug-cc-pV5Z(-DK) and icMRCI/aug-ccpWCV5Z(-DK), respectively, with or without DK.

Fig. 1-4 show all PECs, SOCs, EAMSc and (T)DMCs generated in this study. If the MOLPRO calculations at some geometries did not converge, they were interpolated or extrapolated from the surrounding points as part of the Duo calculations (see below). We used an adaptive $a b$ initio grid consisting of 150 bond lengths ranging from 0.7 to $8 \AA$ with more points around the equilibrium region. The grid points with the corresponding $a b$ initio values (if converged) are included in the supplementary material and also shown in Fig. 1-4. The icMRCI/aug-cc-pV5Z(-DK) calculations have only converged up to about $3.5 \AA$ due to a smaller number of configurations in the internally contacted reference set used.
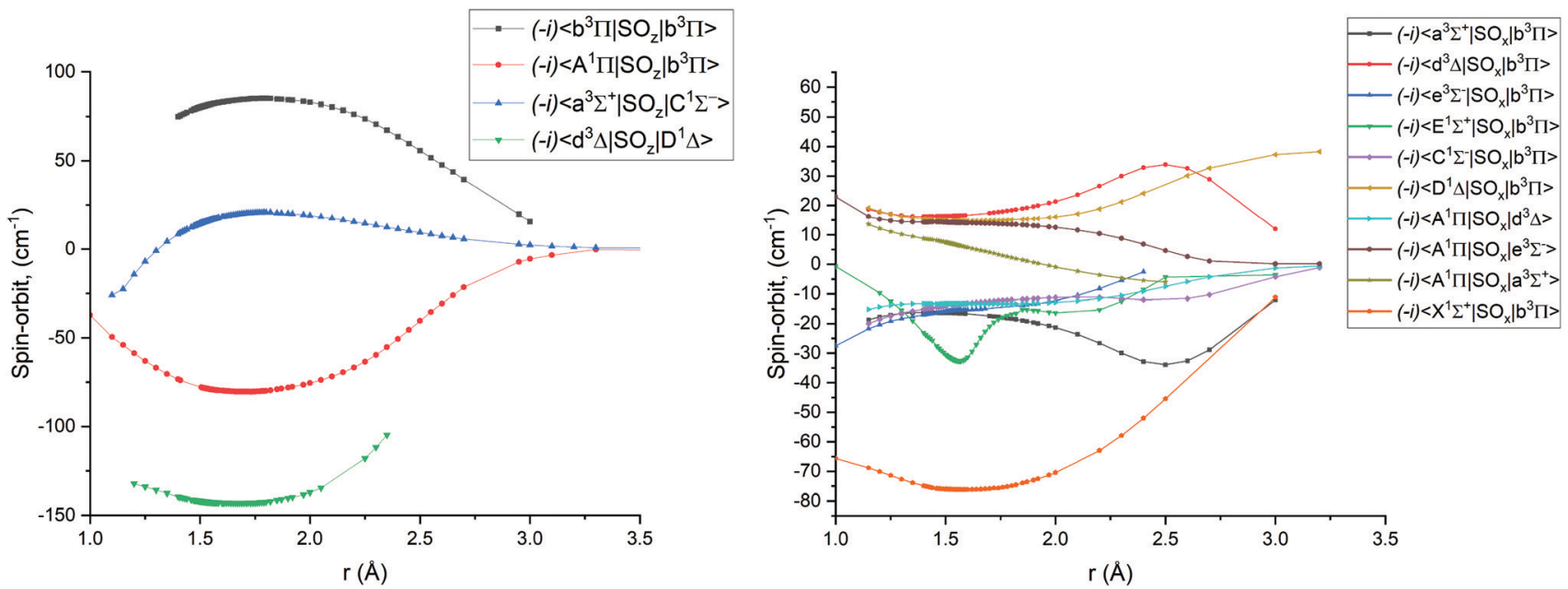

Fig. $2 A b$ initio spin-orbit matrix elements $\left\langle i\left|S_{x}\right| j\right\rangle$ for PN at the icMRCl/aug-cc-pV5Z-DK level of theory. The MOLPRO values of the magnetic quantum numbers $m_{\mathrm{S}}$ for the curves can be found in Table 2 . 


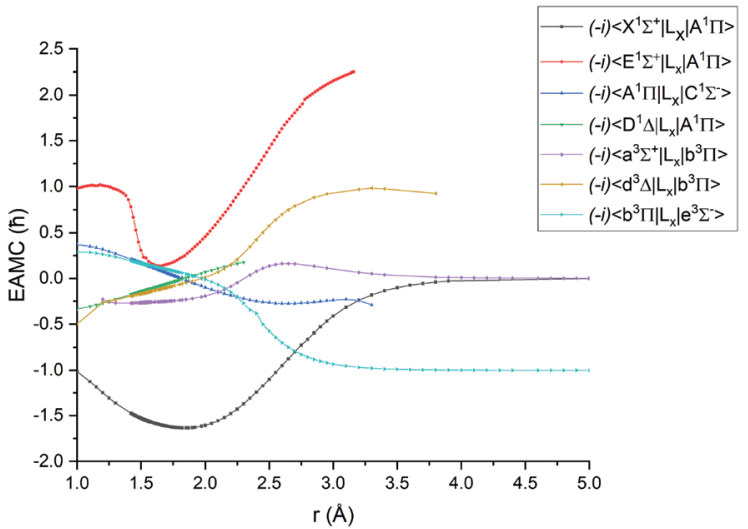

Fig. 3 Ab initio (icMRCI + Q/ECP10MWB) electronic angular momentum couplings for $\mathrm{PN}$ in the units of $\hbar$.
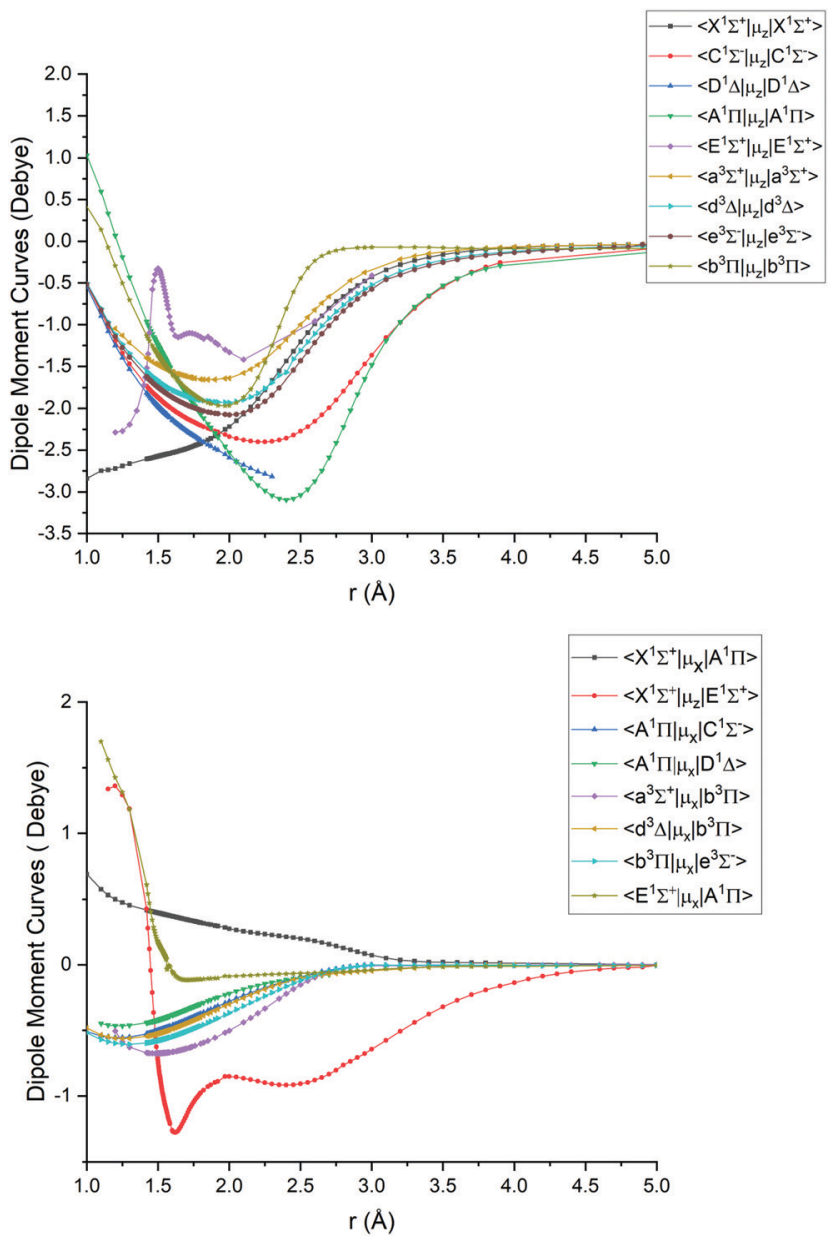

Fig. $4 A$ A initio (icMRCl $+\mathrm{Q} / \mathrm{ECP} 10 \mathrm{MWB}$ ) dipole moment curves for $\mathrm{PN}$ : diagonal (upper), off-diagonal (lower).

\subsection{Duo calculations}

We use the program Duo ${ }^{36,43}$ to solve the coupled Schrödinger equation for 9 lowest electronic states of PN. Duo is a variational program capable of solving rovibronic problems for a general (open-shell) diatomic molecule with an arbitrary number of couplings, see, for example, ref. 44-47. All $a b$ initio couplings between these 9 states are taken into account as described below. The goal of this paper is to build a comprehensive $a b$ initio spectroscopic model for this electronic system of PN based on the icMRCI + Q/ECP10MWB and icMRCI/aug-cc-pV5Z-DK $a b$ initio curves. We therefore do not attempt a systematic refinement of the $a b$ initio curves by fitting to the experiment, which will be the subject of future work. In order to facilitate the comparison with the experimental data, we, however, perform some shifts of the equilibrium energy $T_{\mathrm{e}}$ and bond length $r_{\mathrm{e}}$ values, as described in further detail below.

In Duo calculations, the coupled Schrödinger equations are solved on an equidistant grid of points, in our case 501, with bond lengths $r_{i}$ ranging from $r=0.85$ to $5 \AA$ using the sinc DVR method. ${ }^{48}$ Our $a b$ initio curves are represented by sparser and less extended grids (see above). For the bond length values $r_{i}$ overlapping with the $a b$ initio ranges, the $a b$ initio curves were projected onto the denser Duo grid using the cubic spline interpolation.

The following functional forms were used for extrapolation outside the original $a b$ initio range: ${ }^{36}$

$$
\begin{gathered}
f_{\mathrm{PEC}}^{\text {short }}(r)=A+B / r, \\
f_{\mathrm{TDMC}}^{\text {short }}(r)=A r+B r^{2}, \\
f_{\text {other }}^{\text {short }}(r)=A+B r,
\end{gathered}
$$

for short range and

$$
\begin{gathered}
f_{\mathrm{PEC}}^{\text {long }}(r)=A+B / r^{6} \\
f_{\mathrm{EAMC}}^{\text {long }}(r)=A+B r, \\
f_{\text {other }}^{\text {long }}(r)=A / r^{2}+B / r^{3}
\end{gathered}
$$

for long range, where $A$ and $B$ are stitching parameters.

The vibrational basis set was taken as eigensolutions of the nine uncoupled 1D problems for each PEC. The corresponding basis set constructed from $9 \times 501$ eigenfunctions was then contracted to include about 60-80 vibrational functions from each state, enough to fill the PEC up to the corresponding dissociation energies. This gives a total of 640 states. These vibrational basis functions were then combined with the spherical harmonics for the rotational and electronic spin basis set functions. All calculations were performed for ${ }^{31} \mathrm{P}^{14} \mathrm{~N}$ using atomic masses.

\section{Results and discussion}

\subsection{Results of $a b$ initio calculations}

The lowest 9 singlet and triplet PECs $\left(\mathrm{X}^{1} \Sigma^{+}, \mathrm{A}^{1} \Pi, \mathrm{C}^{1} \Sigma^{-}, \mathrm{D}^{1} \Delta\right.$, $\mathrm{E}^{1} \Sigma^{+}, \mathrm{a}^{3} \Sigma^{+}, \mathrm{b}{ }^{3} \Pi, \mathrm{d}^{3} \Delta, \mathrm{e}^{3} \Sigma^{-}$) calculated at the icMRCI $+\mathrm{Q} /$ ECP10MWB level and are shown in Fig. 1. Table 1 presents spectroscopic constants for these states estimated using the corresponding (spin-orbit-free) PECs and compares to previous studies. ${ }^{17,19,29,33,34,41,42}$ These values agree well for the states $\mathrm{X}^{1} \Sigma^{+}, \mathrm{C}{ }^{1} \Sigma^{-} \mathrm{D}^{1} \Delta, \mathrm{E}^{1} \Sigma^{+}, \mathrm{a}{ }^{3} \Sigma^{+}, \mathrm{e}^{3} \Sigma^{-}, \mathrm{b}{ }^{3} \Pi, \mathrm{d}^{3} \Delta$ as estimated using the corresponding (spin-orbit-free) PECs. For the $\mathrm{E}^{1} \Sigma^{+}$ 
Table 1 Comparison of spectroscopic constants taken from previous works and calculated from our ab initio curves (icMRCI + Q/ECP10MWB): Dissociation energy $D_{\mathrm{e}}$ in $\mathrm{cm}^{-1}$ (rounded to $3 \mathrm{s.f.}$ ), electronic equilibrium energy $T_{\mathrm{e}}$ in $\mathrm{cm}^{-1}$, equilibrium bond length $r_{\mathrm{e}}$ in $\AA$, harmonic constant $\omega$ in $\mathrm{cm}^{-1}$, rotational constant $B_{\mathrm{e}}$ in $\mathrm{cm}^{-1}$

\begin{tabular}{|c|c|c|c|c|c|c|}
\hline State & & $D_{\mathrm{e}}$ & $T_{\mathrm{e}}$ & $r_{\mathrm{e}}$ & $\omega_{\mathrm{e}}$ & $B_{\mathrm{e}}$ \\
\hline \multirow[t]{6}{*}{$\mathrm{X}^{1} \Sigma^{+}$} & This work & 53100.00 & 0.00 & 1.49 & 1303.23 & 0.78 \\
\hline & Expt. $^{33}$ & 50812.91 & 0.00 & 1.4869 & 1337.24 & 0.78549 \\
\hline & Expt. $^{17}$ & 51938.86 & 0.00 & & 1336.992 & 0.78648 \\
\hline & $\mathrm{Calc}^{29}$ & 51454.07 & 0.00 & 1.4918 & 1339.61 & 0.78549 \\
\hline & Calc. $^{41}$ & 50645.15 & 0.00 & 1.4948 & 1333.84 & 0.7823 \\
\hline & Calc. $^{34}$ & 49683.73 & 0.00 & 1.4977 & 1328.21 & 0.77872 \\
\hline \multirow[t]{6}{*}{$A^{1} \Pi$} & This work & 44900.00 & 42143.66 & 1.56 & 1041.07 & 0.71 \\
\hline & Expt. $^{33}$ & 41134.26 & 39688.52 & 1.5424 & 1103.09 & 0.73071 \\
\hline & Expt. $^{19}$ & & 39805.90 & & 1103 & 0.731 \\
\hline & $\mathrm{Calc}^{29}$ & 40995.53 & 40032.55 & 1.5476 & 1104.81 & 0.7306 \\
\hline & Calc. $^{41}$ & 41331.86 & & 1.5501 & 1100.24 & 0.727505 \\
\hline & Calc. $^{34}$ & 40624.26 & 40610.00 & 1.556 & 1078.42 & 0.7213 \\
\hline \multirow[t]{5}{*}{$\mathrm{D}^{1} \Delta$} & This work & 43100.07 & 43287.03 & 1.63 & 1016.44 & 0.66 \\
\hline & Calc. $^{29}$ & 39993.49 & 42106.86 & 1.6073 & 1018.98 & 0.67724 \\
\hline & Calc. $^{42}$ & 33713.96 & 41618.19 & 1.622 & 967.9 & 0.664 \\
\hline & Calc. $^{34}$ & 39448.89 & 41835.96 & 1.614 & 1010.647 & 0.671 \\
\hline & Calc. $^{41}$ & 41555.28 & & 1.6196 & 1002.269 & 0.665056 \\
\hline \multirow[t]{4}{*}{$\mathrm{C}^{1} \Sigma^{-}$} & This work & 45300.00 & 41417.18 & 1.63 & 998.65 & 0.66 \\
\hline & Calc. $^{29}$ & 42766.02 & 39298.72 & 1.6211 & 977.5 & 0.6639 \\
\hline & Calc. $^{42}$ & 37827.38 & 37504.77 & 1.617 & 1108.8 & 0.669 \\
\hline & Calc. $^{34}$ & 41852.09 & 39505.02 & 1.627 & 973.927 & 0.6597 \\
\hline \multirow[t]{2}{*}{$\mathrm{E}^{1} \Sigma^{+}$} & This work & 30200.00 & 56793.82 & 1.86 & 702.48 & 0.50 \\
\hline & Calc. ${ }^{34}$ & 26188.80 & 55103.80 & 1.889 & 743.74 & 0.4893 \\
\hline \multirow[t]{4}{*}{$a^{3} \Sigma^{+}$} & This work & 26600.62 & 26632.70 & 1.66 & 917.51 & 0.63 \\
\hline & Calc. $^{29}$ & 25249.32 & 26065.66 & 1.6481 & 941.61 & 0.64253 \\
\hline & Calc. $^{42}$ & 19599.26 & 24761.21 & 1.669 & 787.4 & 0.628 \\
\hline & Calc. ${ }^{34}$ & 23785.28 & 25817.80 & 1.655 & 913.765 & 0.6375 \\
\hline \multirow[t]{4}{*}{$\mathrm{e}^{3} \Sigma^{-}$} & This work & 32670.00 & 39370.41 & 1.64 & 974.15 & 0.65 \\
\hline & Calc. $^{29}$ & 32081.01 & 37951.02 & 1.624 & 981.37 & 0.66149 \\
\hline & Calc. $^{42}$ & 37827.39 & 37504.77 & 1.617 & 1108.8 & 0.669 \\
\hline & Calc. $^{34}$ & 30665.19 & 37956.44 & 1.632 & 982.255 & 0.6556 \\
\hline \multirow[t]{4}{*}{$\mathrm{d}^{3} \Delta$} & This work & 33300.00 & 34860.41 & 1.65 & 960.01 & 0.65 \\
\hline & Calc. $^{29}$ & 29679.37 & 32975.56 & 1.6324 & 949.99 & 0.65376 \\
\hline & Calc. $^{42}$ & 23551.38 & 32504.13 & 1.666 & 770.3 & 0.629 \\
\hline & Calc. $^{34}$ & 28471.36 & 33125.18 & 1.64 & 959.263 & 0.6495 \\
\hline \multirow[t]{4}{*}{$\mathrm{b}^{3} \Pi$} & This work & 33400.00 & 35146.16 & 1.56 & 1070.81 & 0.71 \\
\hline & Calc. $^{29}$ & 29197.77 & 33671.79 & 1.5449 & 1113.24 & 0.73301 \\
\hline & Calc. $^{42}$ & 21696.31 & 34359.20 & 1.558 & 1124.3 & 0.72 \\
\hline & Calc. ${ }^{34}$ & 27672.87 & 33843.01 & 1.555 & 1102.494 & 0.7224 \\
\hline
\end{tabular}

state, however we note that there are very few studies of that state, so further computational investigation is potentially required. For the $\mathrm{A}^{1} \Pi$ state, the $T_{\mathrm{e}}$ value (the equilibrium electronic energy relative to the minimum value of $\left.\mathrm{X}^{1} \Sigma^{+}\right)$is by about $2300 \mathrm{~cm}^{-1}$ larger than previous experimental and $a b$ initio values, which in turn affects the $D_{\mathrm{e}}$ value. We consider this to be an effect of the ECP method used in the ab initio calculations and we note that there is a similar magnitude shift in $T_{\mathrm{e}}$ of all other states.

The $a b$ initio SOCs, EAMCs and (T)DMCs are shown in Fig. 2-4 respectively, with their phases indicated and the reference distance for phases taken as $1.5 \AA$. For all these curves the post-processing of ab initio calculations included (i) inter- and extrapolations for the missing points using neighboring geometries and (ii) phase mapping using a procedure similar to the one described by Patrascu et al. ${ }^{44}$ Some of the curves also cover only part of the range, which can be seen with the TDMCs $\left\langle\mathrm{X}^{1} \Sigma^{+}\left|\mu_{z}\right| \mathrm{E}^{1} \Sigma^{+}\right\rangle$in Fig. 4 . The rest of the points portrayed incoherent behaviour and hence were
Table 2 MOLPRO magnetic quantum numbers $m_{\mathrm{S}}$ values for the ab initio spin-orbit matrix elements displayed in Fig. 2

\begin{tabular}{lll}
\hline SOC & Bra $m_{\mathrm{S}}$ & Ket $m_{\mathrm{S}}$ \\
\hline$\left\langle\mathrm{A}^{1} \Pi_{y}\left|\mathrm{SO}_{x}\right| \mathrm{d}^{3} \Delta_{x y}\right\rangle$ & 0 & 1 \\
$\left\langle\mathrm{~A}^{1} \Pi_{y}\left|\mathrm{SO}_{x}\right| \mathrm{e}^{3} \Sigma^{-}\right\rangle$ & 0 & 1 \\
$\left\langle\mathrm{a}^{3} \Sigma^{+}\left|\mathrm{SO}_{x}\right| \mathrm{b}^{3} \Pi_{y}\right\rangle$ & 0 & 1 \\
$\left\langle\mathrm{a}^{3} \Sigma^{+}\left|\mathrm{SO}_{x}\right| \mathrm{A}^{1} \Pi_{y}\right\rangle$ & 1 & 0 \\
$\left\langle\mathrm{~d}^{3} \Delta_{x y}\left|\mathrm{SO}_{x}\right| \mathrm{b}^{3} \Pi_{y}\right\rangle$ & 0 & 1 \\
$\left\langle\mathrm{e}^{3} \Sigma^{-}\left|\mathrm{SO}_{x}\right| \mathrm{b}^{3} \Pi_{x}\right\rangle$ & 0 & 1 \\
$\left\langle\mathrm{X}^{1} \Sigma^{+}\left|\mathrm{SO}_{x}\right| \mathrm{b}^{3} \Pi_{y}\right\rangle$ & 0 & 1 \\
$\left\langle\mathrm{E}^{1} \Sigma^{+}\left|\mathrm{SO}_{x}\right| \mathrm{b}^{3} \Pi_{y}\right\rangle$ & 0 & 1 \\
$\left\langle\mathrm{C}^{1} \Sigma^{-}\left|\mathrm{SO}_{x}\right| \mathrm{b}^{3} \Pi_{y}\right\rangle$ & 0 & 1 \\
$\left\langle\mathrm{D}^{1} \Delta_{z}\left|\mathrm{SO}_{x}\right| \mathrm{b}^{3} \Pi_{x}\right\rangle$ & 0 & 1 \\
$\left\langle\mathrm{~b}^{3} \Pi_{x}\left|\mathrm{SO}_{z}\right| \mathrm{b}^{3} \Pi_{x}\right\rangle$ & 1 & 1 \\
$\left\langle\mathrm{~A}^{1} \Pi_{x}\left|\mathrm{SO}_{z}\right| \mathrm{b}^{3} \Pi_{x}\right\rangle$ & 0 & 0 \\
$\left\langle\mathrm{a}^{3} \Sigma^{+}\left|\mathrm{SO}_{z}\right| \mathrm{C}^{1} \Sigma^{-}\right\rangle$ & 0 & 0 \\
$\left\langle\mathrm{~d}^{3} \Delta_{z}\left|\mathrm{SO}_{z}\right| \mathrm{D}^{1} \Delta_{z}\right\rangle$ & 0 & 0
\end{tabular}

dropped in the post-processing. When calculating spectra for curves with dropped points, the lines were extrapolated using cubic splines as part of the Duo calculations.

\subsection{The $\mathrm{A}^{1} \Pi-\mathrm{X}^{1} \Sigma^{+}$band using different levels of $a b$ initio theory}

As the $\mathrm{A}^{1} \Pi-\mathrm{X}^{1} \Sigma^{+}$band is one of the most important spectroscopic systems of PN, here we compare the $\mathrm{X}^{1} \Sigma^{+}$and $\mathrm{A}^{1} \Pi$ PECs and the $\mathrm{A}^{1} \Pi-\mathrm{X}^{1} \Sigma^{+}$TDMC computed using different levels of theory: icMRCI + Q/ECP10MWB, icMRCI/aug-cc-pV5Z-DK and icMRCI/aug-cc-pwCV5Z-DK, see Fig. 5 and 6. As part of the spectroscopic model for PN, even a slight change of these curves can have a profound effect on the simulated spectra or lifetimes.

In Fig. 6, we present a comparison of TDMCs of the $A^{1} \Pi$ and $\mathrm{X}^{1} \Sigma^{+}$states calculated at different levels of theory and results previously calculated by Qin et al. ${ }^{29}$ The TDMC calculated with the ECP10MWB stands out the most with larger values of the dipole moment around the equilibrium (i.e. in the spectroscopically relevant region) and the rest of the ECP-free methods giving curves which lie close to each other. We show below that

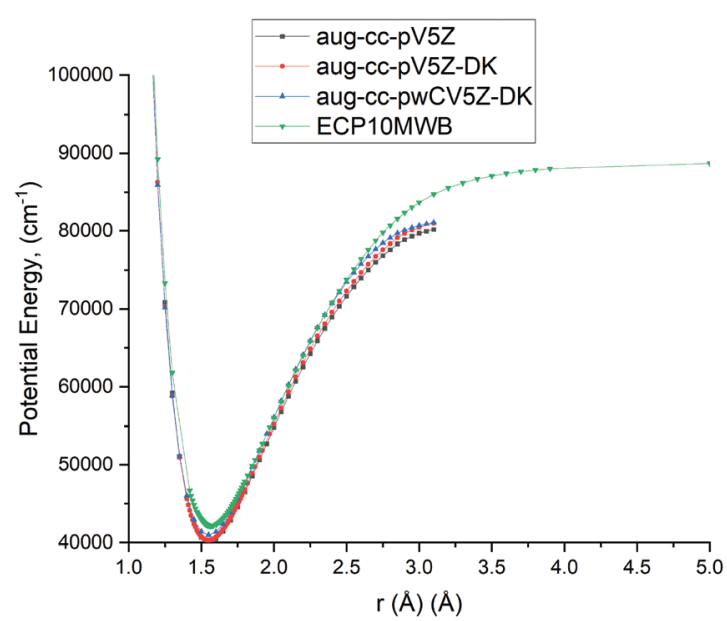

Fig. 5 Comparison of PECs for the $A{ }^{1} \Pi$ state of PN at different levels of theory. 


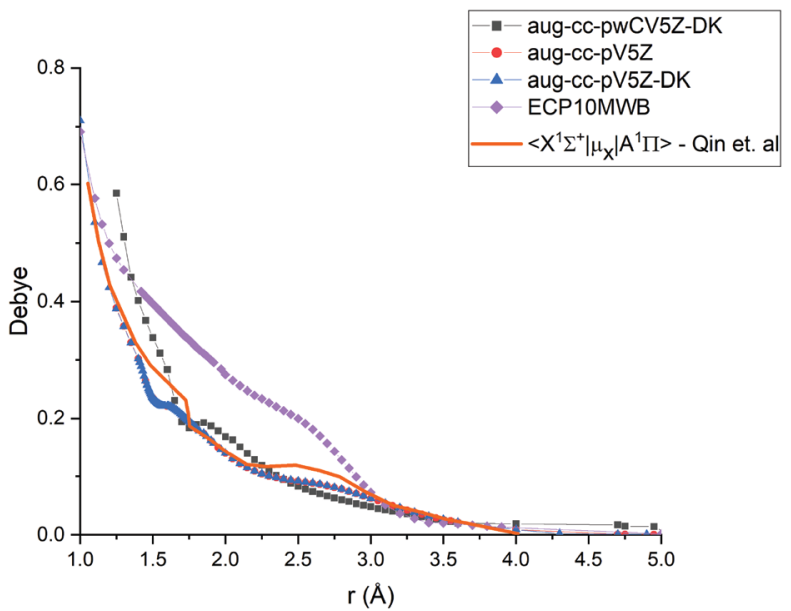

Fig. 6 Comparison of transition dipole moment between $A{ }^{1} \Pi$ and $X^{1} \Sigma^{+}$ states of PN at the different levels of theory (ECP10MWB, icMRCl/aug-CCpV5Z-DK, icMRCl/aug-cc-pWCV5Z-DK) and previously reported results. ${ }^{29}$

the ECP10MWB DMC has improved the A-X lifetime of PN, the only known experimental evidence of the transition probability of this system.

The aug-cc-pWCV5Z-DK and previous results by Qin et al. ${ }^{29}$ show smaller values of TDMC which will lead to weaker intensities and longer lifetimes.

\subsection{Results of Duo calculations}

In this study we work directly with the ab initio data in the grid representation without representing the curves analytically, with exception of the $\mathrm{X}^{1} \Sigma^{+}$state for which we use an empirical potential energy function from Yorke et al. ${ }^{49}$ Using their PEC, we essentially reproduce the $\mathrm{X}^{1} \Sigma^{+}$states ro-vibrational energies of the YYLT line list.

For Duo calculations, we selected the following set of curves: the icMRCI + Q/ECP10MWB PECs shown in Fig. 1 for all but the $\mathrm{X}^{1} \Sigma^{+}$state. The comparison between the $\mathrm{X}^{1} \Sigma^{+} a b$ initio PEC from this work and the empirical PEC from ${ }^{49}$ can be seen in Fig. 7. Similarly for (T)DMCs all curves are ab initio, see Fig. 4, apart from the ground state dipole moment $\left\langle\mathrm{X}^{1} \Sigma^{+}\left|\mu_{z}\right| \mathrm{X}{ }^{1} \Sigma^{+}\right\rangle$,

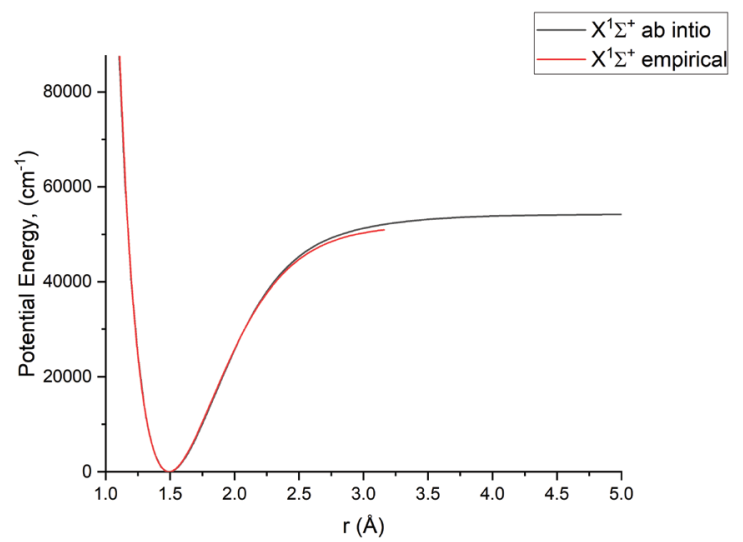

Fig. 7 Comparison between a pseudopotential ab initio $X^{1} \Sigma^{+}$PEC and previously calculated empirical $\mathrm{PEC}^{49}$ of $\mathrm{PN}$. which was also taken from Yorke et $a l .{ }^{49}$ The comparison between two DMCs is shown in Fig. 8. For SOCs, TDMCs and EAMCs we use $a b$ initio obtained curves shown in Fig. 2-4.

The rovibronic wave functions of PN were computed with Duo and then used in conjunction with the $a b$ initio (T)DMCs to produce Einstein $A$ coefficients for all rovibronic transitions between states considered in this work, for the wavenumber range from 0 to $88000 \mathrm{~cm}^{-1}$ and $J \leq 270$. In these calculations, the lower states were capped by the energy threshold $60000 \mathrm{~cm}^{-1}$, which is close to the lowest dissociation threshold, while the upper state threshold was set to $88000 \mathrm{~cm}^{-1}$ (highest asymptotic channel of our nine electronic state system). The line list contains 233418251 transitions between 390368 rovibronic states.

These Einstein $A$ coefficients, organised as per the ExoMol format $^{50}$ in a line list, were then used to calculate lifetimes and spectra. The aforementioned format uses a two file system to represent relevant spectroscopic information, with the energies and specific state quantum numbers included into the States file (.states) and Einstein coefficients appearing in the Transitions file linking different states (.trans). The.states and.trans files produced by Duo are used in conjunction with Exocross $^{51}$ to produce spectra and lifetimes (see below).

\subsection{Partition function}

The partition function of ${ }^{31} \mathrm{P}^{14} \mathrm{~N}$ computed using our $a b$ initio line list is shown in Fig. 9, which is compared to that recently reported by Barklem and Collet. ${ }^{52}$ Since ${ }^{14} \mathrm{~N}$ has a nuclear spin degeneracy of 3 and ${ }^{31} \mathrm{P}$ has nuclear spin degeneracy of 2, we have multiplied Barklem and Collet's partition function by a factor of six to compensate for the different conventions used; we follow ExoMol and HITRAN ${ }^{53}$ and include the full nuclear spin in our partition functions. The differences in higher temperatures can be attributed to incompleteness in the model used by Barklem and Collet. ${ }^{52}$

\subsection{Spectral comparisons}

Using the $a b$ initio ${ }^{31} \mathrm{P}^{14} \mathrm{~N}$ line list, spectral simulations were performed with our code ExoCross. ${ }^{51}$ ExoCross is an open

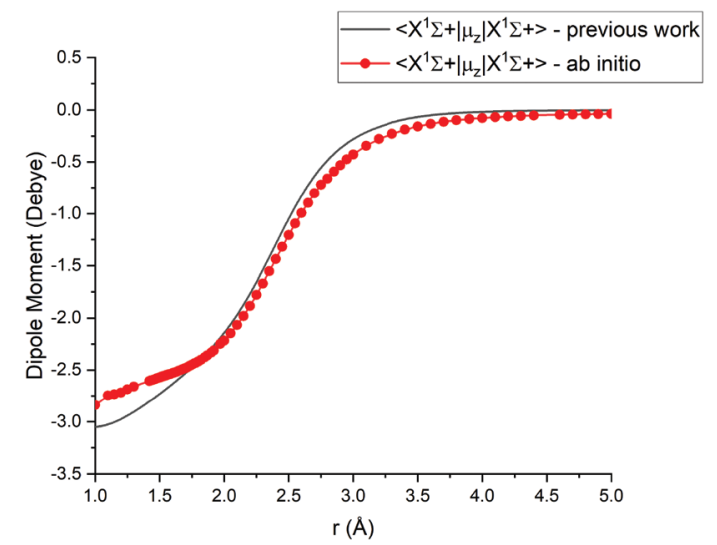

Fig. 8 Comparison between absolute values of the $a b$ initio $X^{1} \Sigma^{+} \mathrm{DMCs}$ from this work (icMRCl + Q/ECP10MWB) and by ${ }^{49}$ (MRCl + Q/aug-CCpCV6Z) 


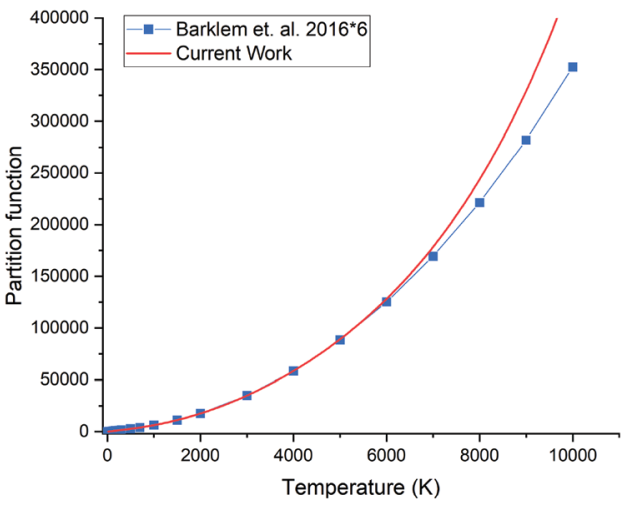

Fig. 9 Comparison of partition functions for ${ }^{31} \mathrm{P}^{14} \mathrm{~N}$ : ab initio this work and the values of Barklem and Collet. ${ }^{52}$

source Fortran 2003 code, see http://exomol.com/software/ or https://github.com/exomol, whose primary use is to produce spectra of molecules at different temperatures and pressures in the form of cross sections using molecular line lists as input. Amongst other features, ExoCross can generate spectra for nonlocal thermal equilibrium conditions characterized with different vibrational and rotational temperatures, lifetimes, Landé $g$-factors, partition and cooling functions.

An overview of the PN absorption spectra in the form of cross sections at the temperature $T=2000 \mathrm{~K}$ is illustrated in Fig. 10. Here, a Gaussian line profile with a half-width-at-halfmaximum (HWHM) of $1 \mathrm{~cm}^{-1}$ was used. This figure shows contributions from each electronic band originating from the ground electronic state. The only band systems that have so far been characterized experimentally are $\mathrm{X}^{1} \Sigma^{+}-\mathrm{X}^{1} \Sigma^{+} \mathrm{A}^{1} \Pi-\mathrm{X}^{1} \Sigma^{+}$, $\mathrm{E}^{1} \Sigma^{+}-\mathrm{X}^{1} \Sigma^{+}$. Here we mainly concentrate on discussing these band systems, due to the lack of experimental detection for other bands calculated in this work.

3.5.1 $\mathrm{X}^{1} \Sigma^{+}$band. The pure rotational $\mathrm{X}$ state transitions represent the main source of the $\mathrm{PN}$ observations in interstellar-medium and stellar spectra. ${ }^{3-10}$ Apart from astrophysical observations, the band has been analysed in multiple

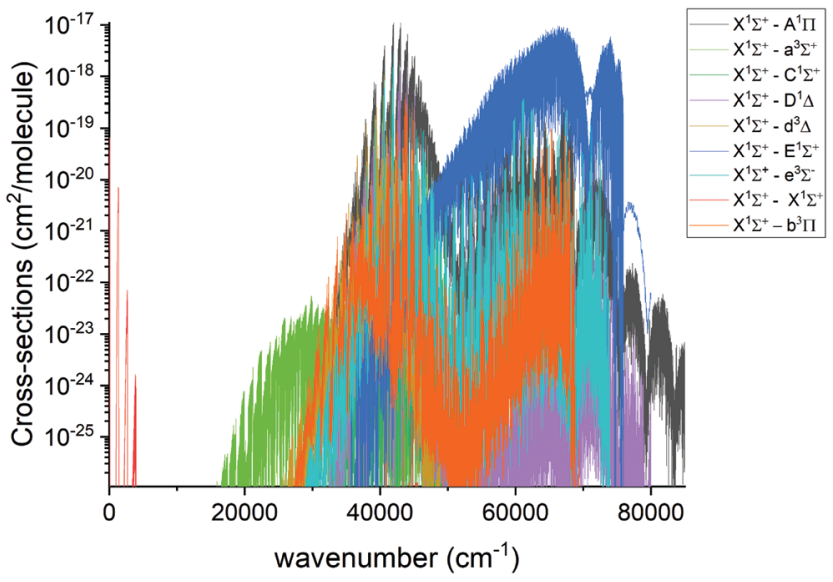

Fig. 10 Overview of the calculated absorption spectra of PN from our model at $T=2000 \mathrm{~K}$. A Gaussian line profile of $\mathrm{HWHM}=1 \mathrm{~cm}^{-1}$ is used. lab experiments as well. ${ }^{14-16,18}$ An overview of the calculated absorption band of PN at $2000 \mathrm{~K}$ is shown in Fig. 11 .

3.5.2 $\mathrm{A}^{1} \boldsymbol{\Pi} \Pi-\mathrm{X}^{1} \Sigma^{+}$band. The visible A-X band system was first observed by Curry et al. ${ }^{33}$ and there have been several subsequent laboratory observations. ${ }^{13,19,21,54}$ Below we compare our results to the experimental spectra; an overview spectrum for the $\mathrm{A}^{1} \Pi-\mathrm{X}^{1} \Sigma^{+}$band system at $2000 \mathrm{~K}$ is given in Fig. 12.

Fig. 13 compares a Duo generated spectrum of the $\mathrm{A}^{1} \Pi-\mathrm{X}^{1} \Sigma^{+}$ $(2,0)$ band to a part of the spectrum from recent experimental observations by Le Floch $e t a l .{ }^{21}$ Even at such a high resolution the main trends of the spectrum are reproduced. However, we have to note a shift of $-2217 \mathrm{~cm}^{-1}$ and some differences in intensities. This will be corrected with a further improvement of our model by fitting the $\mathrm{A}{ }^{1} \Pi$ PEC to the experimental data.

Fig. 14 illustrates a simulated emission $\mathrm{A}^{1} \Pi-\mathrm{X}{ }^{1} \Sigma^{+}$band system at $250 \mathrm{~nm}$, which is compared to the chemiluminescent experimental spectrum of Saraswathy and Krishnamurty, ${ }^{54}$ where we used the rotational temperature setting as $2000 \mathrm{~K}$. However, when trying to adjust this spectrum, it proved to be very sensitive to the $T_{\mathrm{e}}$ and $r_{\mathrm{e}}$ values for the $\mathrm{A}{ }^{1} \Pi$ state. Therefore, for the purpose of this illustration we shifted the

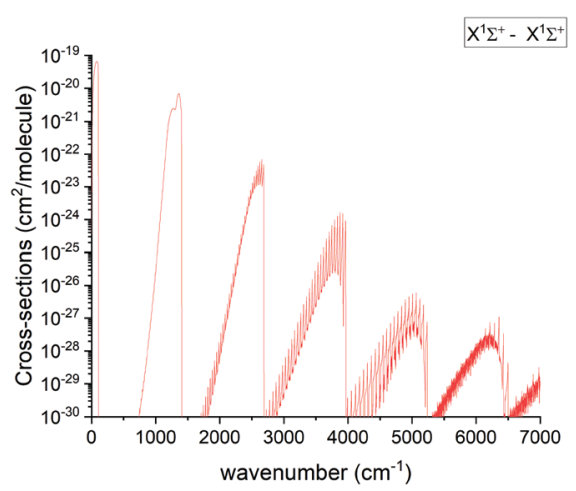

Fig. 11 Overview of the calculated $X^{1} \Sigma^{+}$state rotation-vibration absorption spectrum of PN at $T=2000 \mathrm{~K}$ with a Gaussian line profile of $\mathrm{HWHM}=1 \mathrm{~cm}^{-1}$.

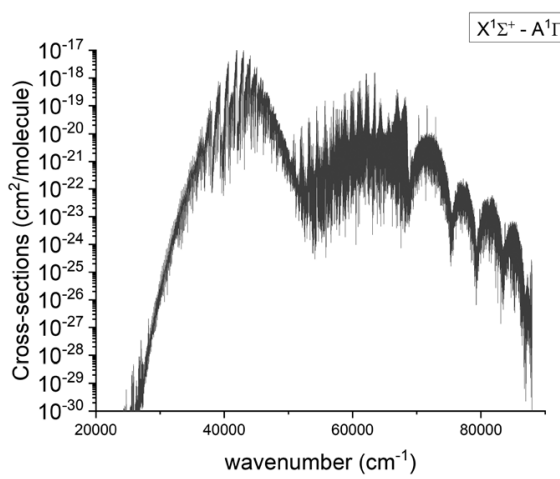

Fig. 12 Calculated absorption spectra of the $A^{1} \Pi-X^{1} \Sigma^{+}$system at $2000 \mathrm{~K}$ with a Gaussian profile of HWHM $=1 \mathrm{~cm}^{-1}$. 


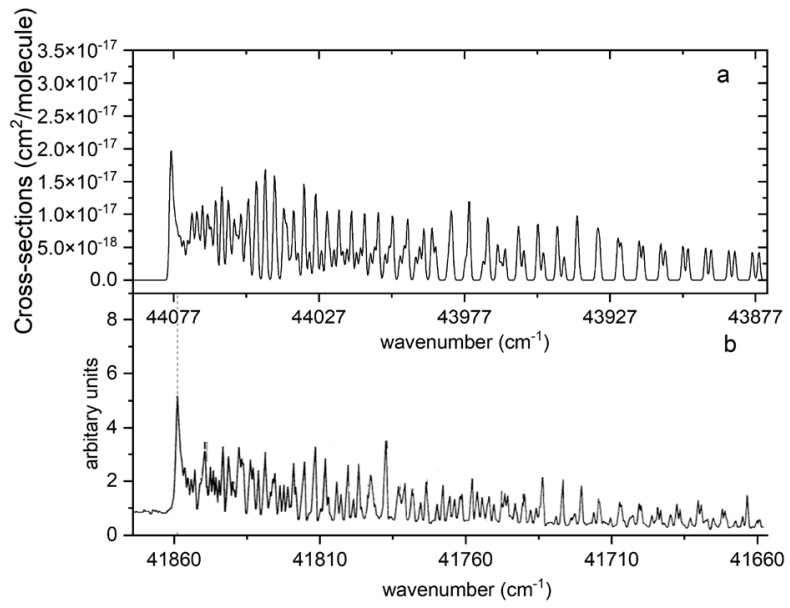

Fig. 13 Comparison of the $A{ }^{1} \Pi-X^{1} \Sigma^{+}(2,0)$ simulated absorption band (panel a) with that recorded by Le Floch et al. ${ }^{21}$ (panel b). The spectrum was simulated at $T=1173.15 \mathrm{~K}$ with a Gaussian profile of $\mathrm{HWHM}=0.5 \mathrm{~cm}^{-1}$ and is offset by $2217 \mathrm{~cm}^{-1}$ so that the features align.

A ${ }^{1} \Pi$ PEC to match experimental values of $T_{\mathrm{e}}$ and $r_{\mathrm{e}}$. There is also a difference in relative magnitudes, which is attributed to the difference in vibrational population distribution of the experiment and our LTE calculations, as there are non-LTE effects which contribute to the spectrum. Even though the PEC was adjusted to the experimental values previously reported by Ghosh et al. ${ }^{19}$ to reproduce this spectrum, there is still a difference of $0.6 \mathrm{~nm}$ between $a b$ initio spectrum and experiment, which we aim to resolve with further improvements of our spectroscopic model via empirical refinements.

Fig. 15 shows a simulated non-LTE absorption $\mathrm{A}^{1} \Pi-\mathrm{X}^{1} \Sigma^{+}$ spectrum of PN to compare to the experiment of Moeller and Silvers. ${ }^{13}$ Most of the major features are again shifted by $\sim 13.5 \mathrm{~nm}$. We attribute the relative difference in strength of

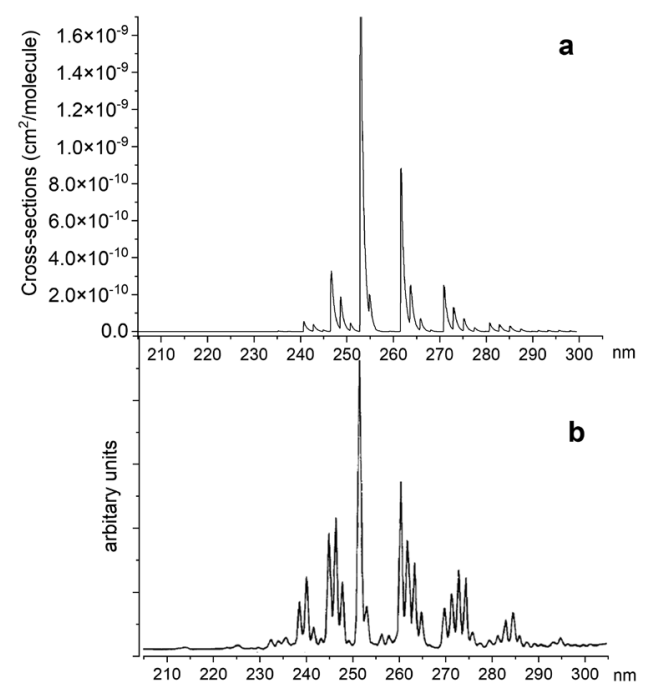

Fig. 14 Comparison of $\mathrm{A}^{1} \Pi-\mathrm{X}^{1} \Sigma^{+}$simulated emission spectra (panel a) with that recorded by Saraswathy and Krishnamurty. ${ }^{54}$ (panel b). The spectra was simulated at $2000 \mathrm{~K}$ rotational temperature with a Gaussian profile and $\mathrm{HWHM}=100 \mathrm{~cm}^{-1}$.

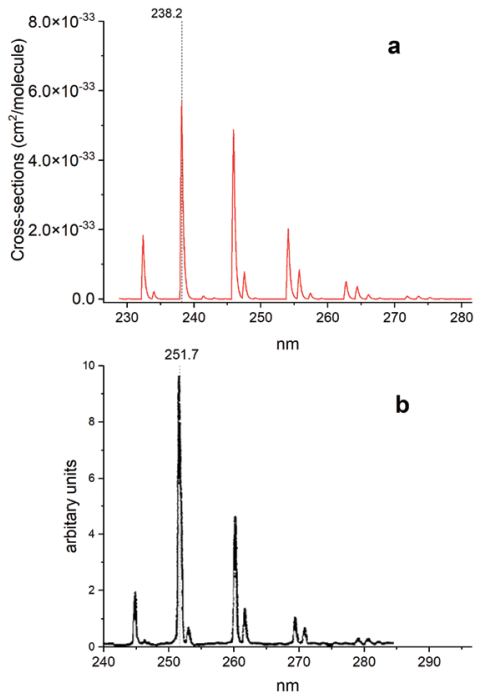

Fig. 15 Comparison of an $A{ }^{1} \Pi-X{ }^{1} \Sigma^{+}$simulated emission spectrum (panel a) with that recorded by Moeller and Silvers. ${ }^{13}$ (panel b). The number at the top indicates the centre of the peak. The spectrum was simulated at $T_{\text {rot }}=500 \mathrm{~K}$ (rotational) and $T_{\text {vib }}=1200 \mathrm{~K}$ (vibrational) temperatures with a Gaussian profile of $\mathrm{HWHM}=100 \mathrm{~cm}^{-1}$.

peaks between theory and experiment to difficulty in reproducing the non-LTE behaviour of the experiment, due to the lack of necessary information in the original paper.

\subsection{Lifetimes}

In this work, lifetimes of PN are calculated using ExoCross, ${ }^{51}$ based on the states and transitions files generated by Duo. ExoCross calculates lifetimes as follows:

$$
\tau_{i}=\frac{1}{\sum_{j<i} A_{i j}}
$$

where $\tau_{i}$ is radiative lifetime, $A_{i j}$ is Einsteins A coefficients, and $i$ and $j$ stand for upper and lower states, respectively. While the lifetimes reported in other works cited below are for vibrational levels and the above formula is for an individual state; however we have found no strong $J$-dependency for the PN lifetimes. This means that $J=0$ does indeed give a good approximation for the vibrational state lifetime. The lack of this dependency can be seen in Fig. 16. The methodology used is described in detail by Tennyson et al..$^{55}$ Fig. 16 shows structures for different vibrational levels, which speaks to the perturbed nature of the A ${ }^{1} \Pi$ state.

The lifetimes of ${ }^{31} \mathrm{P}^{14} \mathrm{~N}$ in the $A{ }^{1} \Pi$ state for $(v=0)$ were measured by Moeller et al., ${ }^{27}$ and also were calculated by Qin $e t a l .{ }^{29}$ and de Brouckere $e t a l^{28}$ While Moeller et $a .^{27}$ report a lifetime of $227 \pm 70 \mathrm{~ns}$ for $\nu^{\prime}=0$ of $\mathrm{A}^{1} \Pi$ our value is only slightly higher at $341 \mathrm{~ns}$, which is closer than previous theoretical calculations of $695.4 \mathrm{~ns}^{29}$ and $742.4 \mathrm{~ns}^{28}$ by a factor of 2 . Table 3 provides a more detailed comparison between our calculated lifetimes and those of Qin et al. ${ }^{29}$ We assume that the major differences in the $\mathrm{A}{ }^{1} \Pi$ lifetimes and previous calculation to the difference in the transition dipole moment 


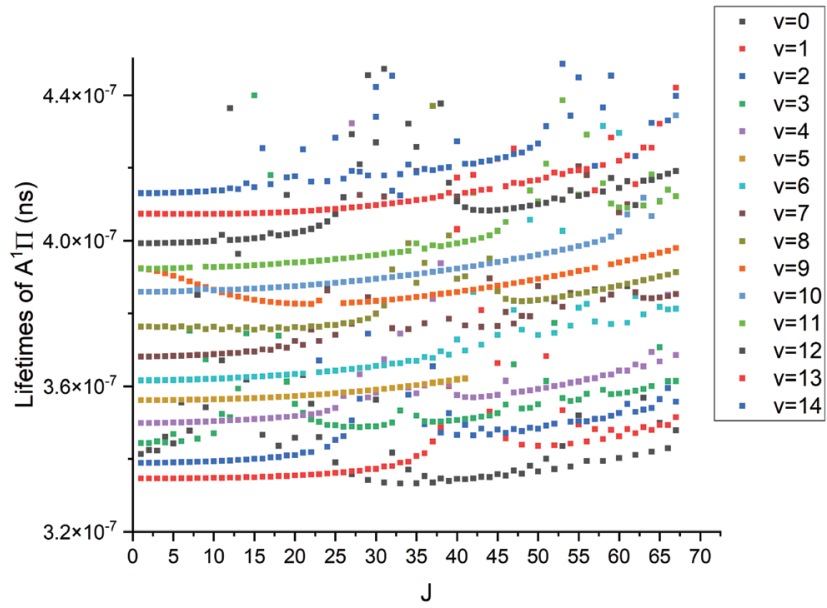

Fig. 16 Lifetimes of $A^{1} \Pi$ state $v s$. J. The top row of points is $v=14$ whereas the bottom is $v=0$.

between $\mathrm{X}^{1} \Sigma^{+}$and $\mathrm{A}^{1} \Pi$ comparison of which can be seen in Fig. 6. In order to check that, we have extracted the $A^{1} \Pi$ TDMC data from the original source ${ }^{29}$ and rerun the lifetime calculation, then getting a comparable result of $677 \mathrm{~ns}$ for $\nu^{\prime}=0$. For the $\mathrm{b}^{3} \Pi$ state, lifetimes seem to be in agreement with Qin et al. ${ }^{29}$ for all but $v=0$, but same cannot be said for the $\mathrm{D}^{1} \Delta$ and $\mathrm{e}^{3} \Sigma^{-}$states. We attribute the bulk of differences in lifetimes for these states to the differences in TDMCs.

Lifetimes for other singlet and triplet states are reported in Table 4. From there we can see that the radiative lifetimes are in milliseconds for $\mathrm{C}^{1} \Sigma^{-}, \mathrm{d}^{3} \Delta$ and a ${ }^{3} \Sigma^{+}$, and nanoseconds for $\mathrm{E}^{1} \Sigma^{+}$. Long lifetimes for the $\mathrm{C}^{1} \Sigma^{-}, \mathrm{d}^{3} \Delta$ and a ${ }^{3} \Sigma^{+}$states are an indication of low probability of transition from these states, leading us to believe that these states would be very difficult to observe in an experiment. This is confirmed with current experimental evidence for PN, as $\mathrm{C}^{1} \Sigma^{-}$, D ${ }^{1} \Delta$, a ${ }^{3} \Sigma^{+}$, d ${ }^{3} \Delta$, e ${ }^{3} \Sigma^{-}$, which have only been observed indirectly through perturbation with the state $\mathrm{A}^{1} \Pi$ by Le Floch et al. ${ }^{21}$ The $\mathrm{b}^{3} \Pi$,

Table 3 Comparison of lifetimes from our current work (A) and Qin et al. ${ }^{29}$ (B). An experimental lifetime $227 \pm 70$ ns was reported by Moeller et al. ${ }^{27}$ for $\nu^{\prime}=0$ of $A^{1} \Pi$

\begin{tabular}{|c|c|c|c|c|c|c|c|c|}
\hline \multirow[b]{2}{*}{$\nu^{\prime}$} & \multicolumn{2}{|c|}{$\mathrm{A}^{1} \Pi / \mathrm{ns}$} & \multicolumn{2}{|c|}{$\mathrm{D}^{1} \Delta / \mu \mathrm{s}$} & \multicolumn{2}{|c|}{$\mathrm{b}^{3} \Pi / \mu \mathrm{s}$} & \multicolumn{2}{|c|}{$\mathrm{e}^{3} \Sigma^{-} / \mu \mathrm{s}$} \\
\hline & A & B & A & B & A & B & A & B \\
\hline 0 & 341.3 & 659.4 & 936.03 & 3872 & 18.76 & 49.2 & 128.51 & 206.9 \\
\hline 1 & 334.6 & 674.3 & 301.51 & 1186 & 15.38 & 27.81 & 52.43 & 98.46 \\
\hline 2 & 338.9 & 660.8 & 120.96 & 615.2 & 13.07 & 19.5 & 73.08 & 59.45 \\
\hline 3 & 344.3 & 646.5 & 110.39 & 378.3 & 11.43 & 15.05 & 61.85 & 40.75 \\
\hline 4 & 349.8 & 642.2 & 88.39 & 246.8 & 10.22 & 12.4 & 53.48 & 30.41 \\
\hline 5 & 356.2 & 643 & 69.23 & 181.8 & 10.06 & 10.62 & 47.30 & 23.68 \\
\hline 6 & 361.6 & 632.1 & 56.85 & 144.3 & 8.55 & 9.364 & 42.60 & 19.26 \\
\hline 7 & 368.1 & 610.6 & 47.37 & 113 & 7.94 & 8.356 & 38.88 & 16.16 \\
\hline 8 & 376.3 & 607.4 & 40.67 & 9.37 & 7.45 & 7.458 & 35.84 & 13.93 \\
\hline 9 & 392.4 & 598.6 & 35.52 & 9.85 & 7.04 & 6.881 & 33.40 & 12.2 \\
\hline 10 & 399.3 & 583.8 & 31.58 & 10.31 & 6.70 & 6.232 & 31.33 & 10.77 \\
\hline 11 & 407.3 & 581.8 & 28.33 & 11.32 & 6.42 & 5.592 & 29.60 & 9.517 \\
\hline 12 & 413.0 & 566.8 & 25.69 & 13.06 & 6.19 & 5.283 & 28.07 & 8.146 \\
\hline 13 & 420.7 & 565.3 & 23.34 & 17.18 & 5.99 & 4.819 & 26.78 & 7.142 \\
\hline 14 & 431.3 & 553.1 & 21.66 & 22.57 & 5.82 & 4.303 & 25.67 & 6.192 \\
\hline
\end{tabular}

Table 4 Calculated lifetimes for the $C^{1} \Sigma^{-}$, a ${ }^{3} \Sigma^{+}$and $d^{3} \Delta E{ }^{1} \Sigma^{+}$states with $\nu^{\prime}$ up to 14. A full list of lifetimes is included in the Exomol states file

\begin{tabular}{lllll}
\hline$\nu^{\prime}$ & $\mathrm{C}^{1} \Sigma^{-} / \mathrm{ms}$ & $\mathrm{a}^{3} \Sigma^{+} / \mathrm{s}$ & $\mathrm{d}^{3} \Delta / \mathrm{ms}$ & $\mathrm{E}^{1} \Sigma^{+} / \mathrm{ns}$ \\
\hline 0 & 65.02 & $2.07 \times 10^{6}$ & 4.19 & 58.70 \\
1 & 53.72 & 9.58 & 9.81 & 52.43 \\
2 & 19.31 & 4.54 & 5.86 & 48.71 \\
3 & 8.21 & 2.85 & 2.77 & 44.25 \\
4 & 4.35 & 2.02 & 1.44 & 39.88 \\
5 & 2.68 & 1.50 & 0.64 & 37.44 \\
6 & 1.83 & 1.18 & 0.03 & 34.03 \\
7 & 1.33 & 0.97 & 0.39 & 30.09 \\
8 & 1.02 & 0.81 & 0.19 & 28.67 \\
9 & 0.81 & 0.69 & 0.35 & 27.89 \\
10 & 0.66 & 0.06 & 0.31 & 27.76 \\
11 & 0.56 & 0.41 & 0.27 & 27.97 \\
12 & 0.48 & 0.33 & 0.23 & 28.23 \\
13 & 0.42 & 0.09 & 0.17 & 28.71 \\
14 & 0.37 & 0.04 & 0.05 & 29.77
\end{tabular}

$\mathrm{d}^{3} \Delta, \mathrm{e}^{3} \Sigma^{-}$states have been also previously identified only through perturbation with $\mathrm{A}^{1} \Pi$ state by Saraswathy and Krishnamurty. ${ }^{20}$

\section{Conclusion}

In this work, a comprehensive ab initio spectroscopic model for the nine lowest electronic states of PN is presented. A full set of potential energy, (transition) dipole moment, spin-orbit coupling, and electronic angular momenta coupling curves for these 9 electronic states was produced $a b$ initio using the icMRCI + Q/ECP10MWB and icMRCI/aug-cc-pV5Z(-DK) methods. These curves were then processed via the Duo program to solve the fully-coupled nuclear-motion Schrödinger equation. Many of the results show satisfactory agreement with previous computational works, but there are certain differences in lifetimes and predicted spectra which indicate that further investigation backed by experimental data is needed to obtain a reliable spectroscopic model. In the next work we aim to tackle such differences, by producing an accurate, empirical line list for ${ }^{31} \mathrm{P}^{14} \mathrm{~N}$ for use in astrophysical spectroscopy of distant stars and exoplanets. In order to achieve this, the $a b$ initio curves will be refined by fitting to the experimental data collected and processed via MARVEL methodology. ${ }^{56}$ Additionally non-adiabatic coupling effects, which are most important for the heavily perturbed $A^{1} \Pi$ state, will be included for finer accuracy. With PN being detected in multiple different interstellar media and phosphorous being a key element to life as we know it, PN is becoming a more important spectroscopic molecule. This work should provide an improved data quality for spectroscopic searches of PN in different astronomical environments, potentially leading to the detection of PN at ultraviolet wavelengths.

The supplementary materials in this paper contain our final spectroscopic model for PN in a form of a Duo input file, an ExoMol States file including lifetimes and a partition function file. This Duo file is ready to be directly used with the Duo program, see http://exomol.com/software/ allowing our results to be reproduced directly. 


\section{Conflicts of interest}

There are no conflicts to declare.

\section{Acknowledgements}

The authors thank Alec Owens for helpful discussions during this project. This work was supported by UK STFC under grant ST/R000476/1. This work made use of the STFC DiRAC HPC facility supported by BIS National E-infrastructure capital grant ST/J005673/1 and STFC grants ST/H008586/1 and ST/ K00333X/1. We thank the European Research Council (ERC) under the European Unions Horizon 2020 research and innovation programme through Advance Grant number 883830. We also want to thank Moscow Witte University for sponsoring the fellowship enabling this research. This work is additionally supported by Khalifa University of Science and Technology under Award No. CIRA-2019-054. Khalifa University High power computer was used for the completion of this work.

\section{Notes and references}

1 M. A. Pasek and D. S. Lauretta, Astrobiology, 2005, 5, 515-535.

2 J. S. Greaves, A. M. S. Richards, W. Bains, P. B. Rimmer, H. Sagawa, D. L. Clements, S. Seager, J. J. Petkowski, C. Sousa-Silva, S. Ranjan, E. Drabek-Maunder, H. J. Fraser, A. Cartwright, I. Mueller-Wodarg, Z. Zhan, P. Friberg, I. Coulson, E. Lee and J. Hoge, Nat. Astron., 2020, 5, 655-664. 3 B. E. Turner and J. Bally, Astrophys. J., 1987, 321, L75-L79.

4 L. M. Ziurys, Astrophys. J., Lett., 1987, 321, L81-L85.

5 B. E. Turner, T. Tsuji, J. Bally, M. Guelin and J. Cernicharo, Astrophys. J., 1990, 365, 569-585.

6 T. Yamaguchi, S. Takano, N. Sakai, T. Sakai, S.-Y. Liu, Y.-N. Su, N. Hirano, S. Takakuwa, Y. Aikawa, H. Nomura and S. Yamamoto, Publ. Astron. Soc. Jpn., 2011, 63, L37-L41.

7 F. Fontani, V. M. Rivilla, P. Caselli, A. Vasyunin and A. Palau, Astrophys. J., Lett., 2016, 822, L30.

8 S. N. Milam, D. T. Halfen, E. D. Tenenbaum, A. J. Apponi, N. J. Woolf and L. M. Ziurys, Astrophys. J., 2008, 684, 618-625.

9 E. De Beck, T. Kaminski, N. A. Patel, K. H. Young, C. A. Gottlieb, K. M. Menten and L. Decin, Astron. Astrophys., 2013, 558, A132.

10 L. M. Ziurys, D. R. Schmidt and J. J. Bernal, Astrophys. J., 2018, 856, 169.

11 J. Curry, L. Herzberg and G. Herzberg, Z. Phys., 1933, 86, 348-366.

$12 \mathrm{M} . \mathrm{Wu}$ and T. P. Fehlner, Chem. Phys. Lett., 1975, 36, 114-116.

13 M. B. Moeller and S. J. Silvers, Chem. Phys. Lett., 1973, 19, 78-81.

14 R. M. Atkins and P. L. Timms, Spectrochim. Acta, Part A, 1977, 33, 853-857.

15 J. Hoeft, E. Tiemann and T. Törring, Z. Naturforsch., A: Phys., Phys. Chem., Kosmophys., 1972, 27, 703-704.
16 F. C. Wyse, W. Gordy and E. L. Manson, J. Chem. Phys., 1972, 57, 1106.

17 G. Cazzoli, L. Cludi and C. Puzzarini, THEOCHEM, 2006, 780-81, 260-267.

18 I. K. Ahmad and P. A. Hamilton, J. Mol. Spectrosc., 1995, 169, 286-291.

19 S. N. Ghosh, R. D. Verma and J. VanderLinde, Can. J. Phys., 1981, 59, 1640-1652.

20 P. Saraswathy and G. Krishnamurty, Pramana, 1987, 29, 53-77.

21 A. C. Le Floch, F. Melen, I. Dubois and H. Bredohl, J. Mol. Spectrosc., 1996, 176, 75-84.

22 B. Coquart and J. C. Prudhomme, J. Phys. B: At., Mol. Opt. Phys., 1980, 13, 2251-2254.

23 B. Coquart and J. C. Prudhomme, J. Mol. Spectrosc., 1981, 87, 75-84.

24 R. D. Verma and S. N. Ghosh, J. Phys. B: At., Mol. Opt. Phys., 1984, 17, 3529-3533.

25 R. D. Verma, S. N. Ghosh and Z. Iqbal, J. Phys. B: At., Mol. Opt. Phys., 1987, 20, 3961-3974.

26 H. Bredohl, I. Dubois, D. Macau-Hercot, F. Remy, J. Breton and J. M. Esteva, J. Mol. Spectrosc., 1992, 156, 292-295.

27 M. B. Moeller, M. R. McKeever and S. J. Silvers, Chem. Phys. Lett., 1975, 31, 398-400.

28 G. de Brouckere, D. Feller and J. J. A. Koot, J. Phys. B: At., Mol. Opt. Phys., 1993, 26, 1915-1934.

29 Z. Qin, J. M. Zhao and L. H. Liu, J. Quant. Spectrosc. Radiat. Transfer, 2019, 227, 47-56.

30 K. A. Gingeric, J. Phys. Chem., 1969, 73, 2734-2741.

31 E. O. Huffman, G. Tarbutton, K. L. Elmore, W. O. Cate, H. K. Walters and G. V. Elmore, J. Am. Chem. Soc., 1954, 76, 6239-6243.

32 O. M. Uy, F. J. Kohl and K. D. Carlson, J. Phys. Chem., 1968, 72, 1611-1616.

33 J. Curry, L. Herzberg and G. Herzberg, J. Chem. Phys., 1933, $1,749$.

34 K. Abbiche, M. Salah, K. Marakchi, O. K. Kabbaj and N. Komiha, Mol. Phys., 2014, 112, 117-126.

35 J. Tennyson and S. N. Yurchenko, Mon. Not. R. Astron. Soc., 2012, 425, 21-33.

36 S. N. Yurchenko, L. Lodi, J. Tennyson and A. V. Stolyarov, Comput. Phys. Commun., 2016, 202, 262-275.

37 H.-J. Werner, P. J. Knowles, F. R. Manby, J. A. Black, K. Doll, A. Heßelmann, D. Kats, A. Köhn, T. Korona, D. A. Kreplin, Q. Ma, T. F. Miller, A. Mitrushchenkov, K. A. Peterson, I. Polyak, G. Rauhut and M. Sibaev, J. Chem. Phys., 2020, 152, 144107.

38 T. H. Dunning, J. Chem. Phys., 1989, 90, 1007-1023.

39 I. S. Lim, P. Schwerdtfeger, B. Metz and H. Stoll, J. Chem. Phys., 2005, 122, 104103.

40 D. E. Woon and T. H. Dunning, J. Chem. Phys., 1993, 98, 1358-1371.

41 J.-M. Wang, J.-F. Sun and D.-H. Shi, Chin. Phys. B, 2010, 19, 49-58.

42 F. Grein and A. Kapur, J. Mol. Spectrosc., 1983, 99, 25-34.

43 J. Tennyson and S. N. Yurchenko, Int. J. Quantum Chem., 2017, 117, 92-103. 
44 A. T. Patrascu, C. Hill, J. Tennyson and S. N. Yurchenko, J. Chem. Phys., 2014, 141, 144312.

45 S. N. Yurchenko, I. Szabo, E. Pyatenko and J. Tennyson, Mon. Not. R. Astron. Soc., 2018, 480, 3397-3411.

46 H.-Y. Li, J. Tennyson and S. N. Yurchenko, Mon. Not. R. Astron. Soc., 2019, 486, 2351-2365.

47 L. K. McKemmish, T. Masseron, J. Hoeijmakers, V. V. PérezMesa, S. L. Grimm, S. N. Yurchenko and J. Tennyson, Mon. Not. R. Astron. Soc., 2019, 488, 2836-2854.

48 R. Guardiola and J. Ros, J. Chem. Phys., 1982, 45, 374-389.

49 L. Yorke, S. N. Yurchenko, L. Lodi and J. Tennyson, Mon. Not. R. Astron. Soc., 2014, 445, 1383-1391.

50 J. Tennyson, S. N. Yurchenko, A. F. Al-Refaie, E. J. Barton, K. L. Chubb, P. A. Coles, S. Diamantopoulou, M. N. Gorman, C. Hill, A. Z. Lam, L. Lodi, L. K. McKemmish, Y. Na, A. Owens, O. L. Polyansky, T. Rivlin, C. Sousa-Silva, D. S. Underwood, A. Yachmenev and E. Zak, J. Mol. Spectrosc., 2016, 327, 73-94.
51 S. N. Yurchenko, A. F. Al-Refaie and J. Tennyson, Astron. Astrophys., 2018, 614, A131.

52 P. S. Barklem and R. Collet, Astron. Astrophys., 2016, 588, A96.

53 R. R. Gamache, C. Roller, E. Lopes, I. E. Gordon, L. S. Rothman, O. L. Polyansky, N. F. Zobov, A. A. Kyuberis, J. Tennyson, S. N. Yurchenko, A. G. Császár, T. Furtenbacher, X. Huang, D. W. Schwenke, T. J. Lee, B. J. Drouin, S. A. Tashkun, V. I. Perevalov and R. V. Kochanov, J. Quant. Spectrosc. Radiat. Transfer, 2017, 203, 70-87.

54 P. Saraswathy and G. Krishnamurty, Pramana, 1987, 29, 53-77.

55 J. Tennyson, K. Hulme, O. K. Naim and S. N. Yurchenko, J. Phys. B: At., Mol. Opt. Phys., 2016, 49, 044002.

56 T. Furtenbacher, A. G. Császár and J. Tennyson, J. Mol. Spectrosc., 2007, 245, 115-125. 